Research Article

\title{
Toxicological Studies of Ethanolic Extract of Oecophylla smaragdina (Weaver ANT) Using Animal Models
}

\author{
Natarajan $\mathrm{P}^{* 1}$, K. Balamurugan ${ }^{2}$, M. Thiruppathi ${ }^{1}$, S. Ramarajan ${ }^{1}$ \\ ${ }^{1}$ Sankaralingam Bhuvaneswari College of Pharmacy, Sivakasi, Tamil Nadu, India. \\ ${ }^{2}$ Associate Professor, Annamalai University, Annamalai Nagar, Tamil Nadu, India. \\ *Corresponding author's E-mail: natarajanmpharm@gmail.com
}

Received: 07-01-2021; Revised: 24-02-2021; Accepted: 03-03-2021; Published on: 20-03-2021.

\section{ABSTRACT}

The present study focused to the acute and subacute toxicity studies of ethanolic extracts of Oecophylla smaragdina (EEOS) using animal model. In the acute study, a single dose of $2000 \mathrm{mg} / \mathrm{kg}$ was administered to albino rat which were observed for $72 \mathrm{~h}$. In subacute toxicity study repeated doses of the EEOS were administered. The rats were received two doses of EEOS $(200 \mathrm{and} 400 \mathrm{mg} / \mathrm{kg}$ p.o. respectively) for a period of 28 days. On the 28 th day of the experiment, blood sample was collected by cervical decapitation after anaesthetizing using xylazine + ketamine $(16+100 \mathrm{mg} / \mathrm{kg}$ i.m. $)$ and it was used to identify the hematological and biochemical analysis. Throughout the study, behavior changes, mortality, body weight, food and water consumption were observed and then sacrificed to remove the liver for histopathological examination. Results: No morbidity and mortality were reported. In sub-acute toxicity study, there is no any significant changes were observed in haematological and biochemical parameters at 200 and $400 \mathrm{mg} / \mathrm{kg} / \mathrm{p}$.o. respectively. Conclusion: It was concluded that no toxicity was observed in ethanolic extracts of Oecophylla smaragdina (EEOS).

Keywords: Oecophylla smaragdina, ethanolic, acute, subacute

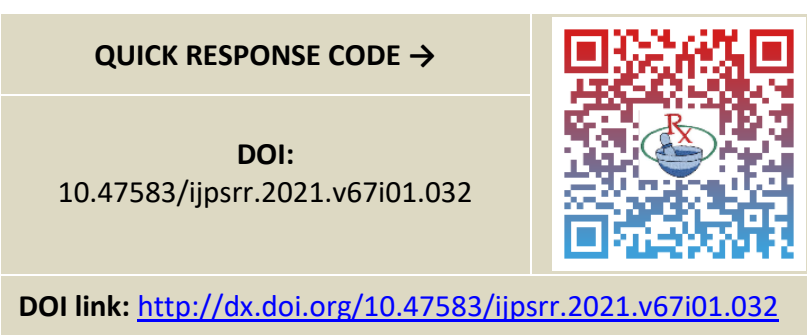

\section{INTRODUCTION}

\section{ntroduction to traditional medicine}

The $21^{\text {st }}$ century is an era in which a tremendous deal of effort and assets are being invested in the research of medicinal plants round the arena. These studies are primarily based in particular on historic, ethnic and sources of information ${ }^{1}$. The usage of animals in conventional medicinal purposes is more and more becoming extra applicable to discussion on conservation biology, public health regulations and sustainable control of natural sources, organic prospection and patents ${ }^{2}$.

In accordance, to the World Health Organization, between 75 and $80 \%$ of the human population uses conventional / traditional folk medicines ${ }^{3}$. It's widely known that the annual worldwide trade in animal-primarily based medicinal products accounts for billions of dollars consistent with year ${ }^{4}$.

Zoo therapy is a form of therapy or remedy which is defined as "the curing of human diseases through use of therapeutic substances obtained or derived from animals". Animals do, indeed, possess an exceptionally wide variety of treatment options that play a significant role inside the curing of humans globally ${ }^{5}$.

The phenomenon of zoo therapy is marked both through a broad geographical distribution and really deep ancient origins. A few authors have shown, animal-based totally drug treatments have been applied due to the fact antiquity ${ }^{6}$. In Latin America as a minimum 584 animal species, disbursed in 13 taxonomic categories, had been utilized in conventional drugs. In India nearly $15-20 \%$ of the Ayurvedic medicines are primarily based on animal derived materials ${ }^{7}$.

The aim of the present research work is to study the toxicological studies of ethanolic extract of Oecophylla smaragdina (OS) (Weaver ant) using animal models.

\section{MAETRIALS AND METHODS}

\section{Taxonomical Identification}

The Oecophylla smaragdina(OS) specimen was identified $\&$ authenticated by Dr. K. Vasudevan, Associate Professor, Department of Zoology, Faculty of Science, Annamalai University, Annamalai nagar, Chidambaram, India. The ant specimen was identified as Oecophylla smaragdina, Family: Formicidae. Voucher Number: OS-001/2016

\section{Extraction method}

The 200g OS were collected and frozen in refrigerator by keeping in polythene covers. The ants were subjected for reflux for 2 hours at $50^{\circ} \mathrm{C}$ using $70 \%$ ethanol by soxhlation ${ }^{8}$. After filtration through Whattman filter paper No. 40, the filtrates were evaporated to dryness in vacuum at $35^{\circ} \mathrm{C}-$ 
$40^{\circ} \mathrm{C}$. The dried EEOS extracts were stored separately in screw cap vial at $4^{\circ} \mathrm{C}$ until further use ${ }^{9}$.

\section{Experimental animals}

Animals of both sex (male \& female) Wistar rats were collected after ethical committee clearance from Sankaralingam Bhuveswari College of Pharmacy, Sivakasi, Virudhunagar dist, Tamil Nadu. The studies were conducted in accordance with the ethical committee (SBCP/2015-2016/CPCSEA/ IAEC/II/I(c). Rats weigh about $125-200 \mathrm{~g} \&$ mice $20-30 \mathrm{~g}$. The animals were maintained in a controlled temp $22 \pm 2^{\circ} \mathrm{C}$ on $12 \mathrm{hr}$ light/dark cycle with free access to standard pellet diet and water ad libitum. After 7 days of acclimatization, the animal was randomly assigned for experimental groups. Each group containing 6 animals were housed individually in labelled cages ${ }^{10}$.

\section{Preparation of formulation}

The EEOS were formulated as suspension in distilled water with $2 \%$ tween 80 as a suspending agent individually. For all the studies freshly, prepared suspensions were used.

\section{Acute toxicity study}

Acute oral toxicity test was performed to identify the LD50 value of EEOS. The experiment was conducted on albino rats. Each group containing 3 animals were used individually. Animals were allowed free access to standard pellet diet (Sai enterprises pvt Ltd, Chennai, India) and water ad libitum. They were maintained in controlled laboratory conditions of $12 \mathrm{hrs}$ dark/light cycle, $22 \pm 2^{\circ} \mathrm{C}$ temperatures and $45-60 \%$ humidity. Any toxic symptoms including mortality and morbidity of the animals were observed.

$\mathrm{LD}_{50}$ study was performed as per OECD-423 guidelines for the EEOS to find out the maximum tolerate dose and minimum lethal dose. The EEOS were given individually, by oral route of rats and the $L D_{50}$ values were calculated. The animals were closely watched for 3 days and were kept for observation up to 14 days to found out delayed mortality.

Animals were fasted prior to administration of dosing. The food was withdrawn overnight and water also withdrawn 3 hrs before drug administration. It is a stepwise procedure, the animals were administrated in the dose of $5 \mathrm{mg} / \mathrm{kg}$ and the dose was increased to 50, 300 and 2000 $\mathrm{mg} / \mathrm{kg}$ p.o. body weight. The mortality of the animals dosed at one step determined the next step. Animals were observed for behavioural changes, toxic symptoms \& mortality up to 3 days and observations were made for 14 days to find out delayed mortality if any. If the animal survived, the second group of animals received a higher dose. If the first animal died or appeared moribund, the second animal received a lower dose ${ }^{11}$.

Animals were observed individually for 3days after dosing at the first 30 minutes, periodically and during the first 24 hrs. Observations include changes in respiration, writhing, tremor, convulsions, hind limb paralysis, sense of touch and sound salivation, urination, diarrhoea and mortality

\section{Sub-acute toxicity studies}

The subacute toxicity procedure was followed by using OECD guidelines 407. Wistar albino rats of both sexes were randomly selected and put into three groups with six rats in each group. Food was withheld overnight but water made available. The animals were weighed prior to administration of the extract. First group served as solvent control (normal saline $10 \mathrm{ml} / \mathrm{kg}$ p.o.) and other groups were administered EEOS at $200 \mathrm{mg}$ and $400 \mathrm{mg} / \mathrm{kg}$ p.o. respectively. The test compounds were given once daily orally for 28 days. All the rats were observed for any physiological and behavioural changes and mortality if any. Organ weight, body weight, food and water consumption were checked every 7 days intervals. All animals were weighed once a week $(1,7,14,21 \& 28$ days). Animals were sacrificed by cervical decapitation under Xylazine + Ketamine $(16+100 \mathrm{mg} / \mathrm{kg}$ i.m.) blood samples were collected, the liver was dissected out for pathological observation $^{12}$.

\section{Gross behavioural studies}

Gross behavioural studies were performed by using effective dose of 200 and $400 \mathrm{mg} / \mathrm{kg} /$ oral of EEOS respectively in rats and following parameters like CNS, reflexes effect, ANS, effect after manipulations were observed $^{13}$.

\section{Haematological analysis}

At the end of subacute toxicity studies experimental period, all surviving animals were fasted overnight. After anaesthetized with Xylazine + Ketamine $(16+100 \mathrm{mg} / \mathrm{kg}$ i.m.), the Blood samples were collected retro-orbital sinus into heparinized tube for the analysis of haematological parameters like haemoglobin, total W.B.C count, total R.B.C count and platelet count.

\section{RESULTS AND DISCUSSION}

Table 1: Acute toxicity in rat treated with EEOS

\begin{tabular}{|c|c|c|c|c|c|c|}
\hline \multicolumn{1}{|c|}{ Observation } & \multicolumn{3}{|c|}{ Effect } \\
\hline Gross behavior activity & $\mathbf{1 h r}$ & $\mathbf{4}$ hrs & $\mathbf{2 4} \mathbf{h r s}$ & $\mathbf{4 8}$ hrs & $\mathbf{7}$ days & $\mathbf{1 4 d a y s}$ \\
\hline Respiration & N & N & N & N & N \\
\hline Writhing & - & - & - & - & - \\
\hline Tremor & - & - & - & - & - \\
\hline Convulsions & - & - & - & - & - \\
\hline Hind limb paralysis & - & - & - & - \\
\hline
\end{tabular}




\begin{tabular}{|c|c|c|c|c|c|c|}
\hline Sense of touch and sound & N & N & N & N & N & N \\
\hline Salivation & N & N & N & N & N & N \\
\hline Urination & N & N & N & N & N & N \\
\hline Diarrhoea & - & - & - & - & - & - \\
\hline Mortality & - & - & - & - & - & - \\
\hline
\end{tabular}

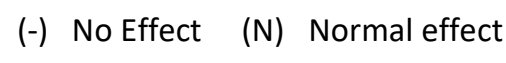

From the acute toxicity study as shown in table 1, it was observed the EEOS at the dose of $2000 \mathrm{mg} / \mathrm{kg}$ p.o. body weight in rats does not cause moribund and mortality.

From the acute toxicity studies as per the OECD 423 guidelines in rat the $L D_{50}$ values was found to be $2000 \mathrm{mg} / \mathrm{kg}$ p.o. body weight. At this dose level no change of respiration, writhing, tremor, convulsions, hind limb paralysis, sense of touch and sound salivation, urination, diarrhoea and mortality. Skin, fur, eyes, mucous membrane and behaviour pattern were normal. There was no moribund or mortality up to the dose level $2000 \mathrm{mg} / \mathrm{kg}$ p.o. and the animals were alive up to the end of the study. The results were showed in table:1

\section{SUB-ACUTE: Repeated dose 28-days oral toxicity studies}

Table 2: Gross behaviour activity (200mg/kg/oral)

\begin{tabular}{|c|c|c|c|c|c|c|c|c|c|c|c|c|c|}
\hline \multirow{2}{*}{ S. No } & \multirow{2}{*}{ Effect on CNS } & \multicolumn{12}{|c|}{ Time (hrs) } \\
\hline & & $1 / 2$ & 1 & $11 / 2$ & 2 & $21 / 2$ & 3 & $31 / 2$ & 4 & 5 & 6 & 12 & 24 \\
\hline 1 & $\frac{\text { Spontaneous motor activity. }}{\text { Restlessness }}$ & - & - & - & - & - & - & - & - & - & - & - & - \\
\hline 2 & Ataxic gait. & - & - & - & - & - & - & - & - & - & - & - & - \\
\hline 3 & Lying flat on the belly & - & - & - & - & - & - & - & - & - & - & - & - \\
\hline 4 & Grooming behaviour & - & - & - & - & - & - & - & - & - & - & - & - \\
\hline 5 & Lying flat on the side & - & - & - & - & - & - & - & - & - & - & - & - \\
\hline 6 & Lying flat on the back & - & - & - & - & - & - & - & - & - & - & - & - \\
\hline 7 & Sleeping & - & - & - & - & - & - & - & - & - & \pm & \pm & - \\
\hline 8 & Narcosis & - & - & - & - & - & - & - & - & - & - & - & - \\
\hline 9 & Bizarre behaviour & - & - & - & - & - & - & - & - & - & - & - & - \\
\hline 10 & Timidity & + & + & + & + & + & + & + & + & + & + & + & + \\
\hline 11 & Straub's phenomenon & - & - & - & - & - & - & - & - & - & - & - & - \\
\hline 12 & Writhing & - & - & - & - & - & - & - & - & - & - & - & - \\
\hline 13 & Tremors & - & - & - & - & - & - & - & - & - & - & - & - \\
\hline 14 & Twitches & - & - & - & - & - & - & - & - & - & - & - & - \\
\hline 15 & Opisthotonus & - & - & - & - & - & - & - & - & - & - & - & - \\
\hline 16 & Chronic convulsions & - & - & - & - & - & - & - & - & - & - & - & - \\
\hline 17 & Tonic convulsions & - & - & - & - & - & - & - & - & - & - & - & - \\
\hline 18 & Rolling and jumping & - & - & - & - & - & - & - & - & - & - & - & - \\
\hline 19 & Convulsions & - & - & - & - & - & - & - & - & - & - & - & - \\
\hline 20 & $\frac{\text { Effect on reflexes: }}{\text { Pinna reflex }}$ & - & - & - & - & - & - & - & - & - & - & - & - \\
\hline 21 & Corneal reflexes & - & - & - & - & - & - & - & - & - & - & - & - \\
\hline 22 & Pain following stimulation & - & - & - & - & - & - & - & - & - & - & - & - \\
\hline 23 & $\begin{array}{c}\text { Effect on autonomic nervous } \\
\text { system: Pupil diameter }\end{array}$ & - & - & - & - & - & - & - & - & - & - & - & - \\
\hline 24 & $\begin{array}{l}\text { Eyelids (closure/ } \\
\text { exophthalamus) }\end{array}$ & - & - & - & - & - & - & - & - & - & - & - & - \\
\hline 25 & Secretion of sweat & - & - & - & - & - & - & - & - & - & - & - & - \\
\hline 26 & Salivation & - & - & - & - & - & - & - & - & - & - & - & - \\
\hline 27 & Lacrimation & - & - & - & - & - & - & - & - & - & - & - & - \\
\hline 28 & Cyanosis & - & - & - & - & - & - & - & - & - & - & - & - \\
\hline 29 & Piloerection & - & - & - & - & - & - & - & - & - & - & - & - \\
\hline 30 & Defecation & - & - & - & - & - & - & - & - & - & - & - & - \\
\hline
\end{tabular}




\begin{tabular}{|c|c|c|c|c|c|c|c|c|c|c|c|c|c|}
\hline 31 & Urination & - & - & - & - & - & - & - & - & - & - & - & - \\
\hline 32 & $\frac{\text { Effect after manipulations: }}{\text { Auditory stimulus response }}$ & + & + & + & + & + & + & + & + & + & + & + & + \\
\hline 33 & Escape after touch & + & + & + & + & + & + & + & + & + & + & + & + \\
\hline 34 & Writhing reflex & - & - & - & - & - & - & - & - & - & - & - & - \\
\hline 35 & Paralysis of hind limbs & - & - & - & - & - & - & - & - & - & - & - & - \\
\hline 36 & Paralysis of fore paws & - & - & - & - & - & - & - & - & - & - & - & - \\
\hline 37 & Catalepsy in induced position & - & - & - & - & - & - & - & - & - & - & - & - \\
\hline
\end{tabular}

Table 3: Gross behaviour activity $(400 \mathrm{mg} / \mathrm{kg} / \mathrm{oral})$

Gross behaviour activity in rat $400 \mathrm{mg} / \mathrm{kg} /$ oral body weight.

\begin{tabular}{|c|c|c|c|c|c|c|c|c|c|c|c|c|c|}
\hline \multicolumn{14}{|c|}{ Gross behaviour activity in rat $400 \mathrm{mg} / \mathrm{kg} /$ oral body weight. } \\
\hline \multirow{2}{*}{ S. No } & \multirow{2}{*}{ Effect on CNS } & \multicolumn{12}{|c|}{ Time (hrs) } \\
\hline & & $1 / 2$ & 1 & $1 \frac{1}{2}$ & 2 & $2 \frac{1}{2}$ & 3 & $3 \frac{1}{2}$ & 4 & 5 & 6 & 12 & 24 \\
\hline 1 & $\frac{\text { Spontaneous motor activity. }}{\text { Restlessness }}$ & - & - & - & - & - & - & - & - & - & - & - & - \\
\hline 2 & Ataxic gait. & - & - & - & - & - & - & - & - & - & - & - & - \\
\hline 3 & Lying flat on the belly & - & - & - & - & - & - & - & - & - & - & - & - \\
\hline 4 & Grooming behaviour & - & - & - & - & - & - & - & - & - & - & - & - \\
\hline 5 & Lying flat on the side & - & - & - & - & - & - & - & - & - & - & - & - \\
\hline 6 & Lying flat on the back & - & - & - & - & - & - & - & - & - & - & - & - \\
\hline 7 & Sleeping & - & - & - & - & - & - & - & - & - & \pm & \pm & - \\
\hline 8 & Narcosis & - & - & - & - & - & - & - & - & - & - & - & - \\
\hline 9 & Bizarre behaviour & - & - & - & - & - & - & - & - & - & - & - & - \\
\hline 10 & Timidity & + & + & + & + & + & + & + & + & + & + & + & + \\
\hline 11 & Straub's phenomenon & - & - & - & - & - & - & - & - & - & - & - & - \\
\hline 12 & Writhing & - & - & - & - & - & - & - & - & - & - & - & - \\
\hline 13 & Tremors & - & - & - & - & - & - & - & - & - & - & - & - \\
\hline 14 & Twitches & - & - & - & - & - & - & - & - & - & - & - & - \\
\hline 15 & Opisthotonus & - & - & - & - & - & - & - & - & - & - & - & - \\
\hline 16 & Chronic convulsions & - & - & - & - & - & - & - & - & - & - & - & - \\
\hline 17 & Tonic convulsions & - & - & - & - & - & - & - & - & - & - & - & - \\
\hline 18 & Rolling and jumping & - & - & - & - & - & - & - & - & - & - & - & - \\
\hline 19 & Convulsions & - & - & - & - & - & - & - & - & - & - & - & - \\
\hline 20 & $\frac{\text { Effect on reflexes: }}{\text { Pinna reflex }}$ & - & - & - & - & - & - & - & - & - & - & - & - \\
\hline 21 & Corneal reflexes & - & - & - & - & - & - & - & - & - & - & - & - \\
\hline 22 & Pain following stimulation & - & - & - & - & - & - & - & - & - & - & - & - \\
\hline 23 & $\begin{array}{l}\text { Effect on autonomic nervous system: } \\
\text { Pupil diameter }\end{array}$ & - & - & - & - & - & - & - & - & - & - & - & - \\
\hline 24 & $\begin{array}{l}\text { Eyelids (closure/ } \\
\text { exophthalamus) }\end{array}$ & - & - & - & - & - & - & - & - & - & - & - & - \\
\hline 25 & Secretion of sweat & - & - & - & - & - & - & - & - & - & - & - & - \\
\hline 26 & Salivation & - & - & - & - & - & - & - & - & - & - & - & - \\
\hline 27 & Lacrimation & - & - & - & - & - & - & - & - & - & - & - & - \\
\hline 28 & Cyanosis & - & - & - & - & - & - & - & - & - & - & - & - \\
\hline 29 & Piloerection & - & - & - & - & - & - & - & - & - & - & - & - \\
\hline 30 & Defecation & - & - & - & - & - & - & - & - & - & - & - & - \\
\hline 31 & Urination & - & - & - & - & - & - & - & - & - & - & - & - \\
\hline
\end{tabular}




\begin{tabular}{|c|c|c|c|c|c|c|c|c|c|c|c|c|c|}
\hline 32 & $\frac{\text { Effect after manipulations: }}{\text { Auditory stimulus response }}$ & + & + & + & + & + & + & + & + & + & + & + & + \\
\hline 33 & Escape after touch & + & + & + & + & + & + & + & + & + & + & + & + \\
\hline 34 & Writhing reflex & - & - & - & - & - & - & - & - & - & - & - & - \\
\hline 35 & Paralysis of hind limbs & - & - & - & - & - & - & - & - & - & - & - & - \\
\hline 36 & Paralysis of fore paws & - & - & - & - & - & - & - & - & - & - & - & - \\
\hline 37 & Catalepsy in induced position & - & - & - & - & - & - & - & - & - & - & - & - \\
\hline
\end{tabular}

Table 4: Results of Organ weight parameters of EEOS treated in rats

\begin{tabular}{|c|c|c|c|c|c|c|}
\hline SI. No & Drug Treatment & Brain (g) & Heart (g) & Kidney(g) & Liver (g) & Stomach(g) \\
\hline $\mathbf{1}$ & $\begin{array}{c}\text { Group l- Control } \\
\text { (Normal saline } 2 \mathrm{ml} / \mathrm{kg} \mathrm{p.o.)}\end{array}$ & $1.52 \pm 0.01$ & $0.65 \pm 0.02$ & $1.11 \pm 0.01$ & $4.32 \pm 0.11$ & $2.21 \pm 0.05$ \\
\hline $\mathbf{2}$ & Group II (EEOS $200 \mathrm{mg} / \mathrm{kg} \mathrm{p.o)}$ & $1.55 \pm 0.01$ & $0.68 \pm 0.03$ & $1.09 \pm 0.05$ & $4.24 \pm 0.16$ & $2.29 \pm 0.012$ \\
\hline $\mathbf{3}$ & Group II (EEOS $400 \mathrm{mg} / \mathrm{kg} \mathrm{p.o)}$ & $1.53 \pm 0.02$ & $0.67 \pm 0.01$ & $1.13 \pm 0.07$ & $4.36 \pm 0.21$ & $2.20 \pm 0.04$ \\
\hline
\end{tabular}

Values are mean \pm SEM; $n=6$ in each group; Group -II \& Group III was compared with Group -I. The values of various organ weights rats were not altered significantly.

Table 5: Results of body weight parameter of EEOS treated in rats

\begin{tabular}{|c|c|c|c|c|c|c|}
\hline \multirow{2}{*}{ SI. No } & \multirow{2}{*}{ Animal treatment } & \multicolumn{5}{|c|}{ Body weight (g) } \\
\hline & & $1^{\text {st }}$ day & $7^{\text {th }}$ day & $14^{\text {th }}$ day & $21^{\text {st }}$ day & $28^{\text {th }}$ day \\
\hline 1 & Group I- Control (Normal saline $2 \mathrm{ml} / \mathrm{kg}$ p.o.) & $151.5 \pm 2.17$ & $159.7 \pm 2.10$ & $154.2 \pm 1.34$ & $158.3 \pm 1.32$ & $153.1 \pm 1.12$ \\
\hline 2 & Group II (EEOS 200 mg/kg p.o) & $150.5 \pm 2.17$ & $155.5 \pm 3.57$ & $157.2 \pm 3.24$ & $162.1 \pm 1.51$ & $166.1 \pm 1.32$ \\
\hline 3 & Group III-(EEOS 400 mg/kg p.o) & $156.2 \pm 2.11$ & $160.2 \pm 1.23$ & $164.6 \pm 1.35$ & $166.7 \pm 2.32$ & $168.3 \pm 2.17$ \\
\hline
\end{tabular}

Values are mean \pm SEM; $n=6$ in each group; Group -II \& Group III was compared with Group -I. The values of body weights rats were not altered significantly.

Table 6: Results of food intake of EEOS treated in rats

\begin{tabular}{|c|c|c|c|c|c|c|}
\hline & & \multicolumn{4}{|c|}{ Food intake $\mathbf{g})$} \\
\hline $\mathbf{1}$ & Animal treatment & $\mathbf{7}^{\text {th }}$ day & $\mathbf{1 4}^{\text {th }}$ day & $\mathbf{2 1}^{\text {st }}$ day & $\mathbf{2 8}^{\text {th }}$ day \\
\hline $\mathbf{2}$ & Group I- Control (Normal saline $2 \mathrm{ml} / \mathrm{kg} \mathrm{p.o.)}$ & $41.63 \pm 0.46$ & $43.24 \pm 0.54$ & $47.15 \pm 0.67$ & $49.14 \pm 0.47$ \\
\hline $\mathbf{3}$ & Group II - (EEOS $200 \mathrm{mg} / \mathrm{kg} \mathrm{p.o)}$ & $40.12 \pm 0.34$ & $43.34 \pm 0.41$ & $44.43 \pm 0.48$ & $46.07 \pm 0.56$ \\
\hline
\end{tabular}

Values are mean \pm SEM; $n=6$ in each group; Group -II \& Group III was compared with Group -I. The values of food intake rats were not altered significantly

Table 7: Results of water intake of EEOS treated in rats

\begin{tabular}{|c|c|c|c|c|c|c|}
\hline & \multicolumn{4}{|c}{ Water Intake (ml) } \\
\hline & SL. No & Animal treatment & $\mathbf{7}^{\text {th }}$ day & $\mathbf{1 4}^{\text {th }}$ day & $\mathbf{2 1}^{\text {st }}$ day & $\mathbf{2 8}^{\text {th }}$ day \\
\hline 1 & Group I- Control (Normal saline $2 \mathrm{ml} / \mathrm{kg}$ p.o.) & $54.32 \pm 2.32$ & $53.21 \pm 1.86$ & $49.54 \pm 2.53$ & $51.45 \pm 2.12$ \\
\hline 2 & Group II - EEOS $200 \mathrm{mg} / \mathrm{kg} \mathrm{p.o)}$ & $50.25 \pm 1.25$ & $49.36 \pm 1.56$ & $51.78 \pm 2.03$ & $51.31 \pm 1.96$ \\
\hline 3 & Group III - EEOS $400 \mathrm{mg} / \mathrm{kg}$ p.o) & $55.74 \pm 2.56$ & $56.41 \pm 2.97$ & $54.22 \pm 2.24$ & $55.64 \pm 2.14$ \\
\hline
\end{tabular}

Values are mean \pm SEM; $n=6$ in each group; Group -II \& Group III was compared with Group -I. The values of water intake rats were not altered significantly.

The results of the subacute toxicity for various organ weight, body weight, food intake and water intake were shown in the table- 4,5,6 and7. The weight of various organ weight, body weight, food intake and water intake for
EEOS treated groups at 200 and $400 \mathrm{mg} / \mathrm{kg}$ p.o. respectively were not altered significantly when compared to normal control group. 
Table 8: Results of haematological parameters of EEOS treated in rats

\begin{tabular}{|c|c|c|c|c|c|}
\hline SI. No & Animal treatment & Haemoglobin gm $\%$ & $\begin{array}{c}\text { WBC } \\
\left(\times 10^{3} / \text { cu.mm }\right)\end{array}$ & $\begin{array}{c}\text { RBC } \\
\left(\times 10^{6} / \text { cu.mm }\right)\end{array}$ & $\begin{array}{c}\text { Platelet } \\
\left(\times 10^{3} / \mu l\right)\end{array}$ \\
\hline 1 & Group I- Control (Normal saline $2 \mathrm{ml} / \mathrm{kg} \mathrm{p.o.)}$ & $14.24 \pm 0.07$ & $8.26 \pm 0.05$ & $4.96 \pm 0.06$ & $5.68 \pm 0.07$ \\
\hline 2 & Group II - EEOS $200 \mathrm{mg} / \mathrm{kg} \mathrm{p.o)}$ & $14.47 \pm 0.11$ & $8.96 \pm 0.11$ & $4.31 \pm 0.09$ & $5.71 \pm 0.08$ \\
\hline 3 & Group III - EEOS $400 \mathrm{mg} / \mathrm{kg} \mathrm{p.0)}$ & $14.31 \pm 0.09$ & $8.44 \pm 0.25$ & $4.48 \pm 0.08$ & $6.37 \pm 0.08$ \\
\hline
\end{tabular}

Values are mean \pm SEM; n=6 in each group; Group -II \& Group III was compared with Group -I. The values of haematological parameters rats were not altered significantly.

The haematological parameters of EEOS like Haemoglobin, WBC, RBC and Platelet were not significant change when compared to Control group. The results of haematological parameters were mentioned in table 8 .

Table 9: Results of biochemical parameters of EEOS treated in rats

\begin{tabular}{|c|c|c|c|c|c|}
\hline SI. No & Animal treatment & $\begin{array}{c}\text { Glucose } \\
\text { (mg/dL) }\end{array}$ & $\begin{array}{c}\text { Urea } \\
(\mathbf{m m o l} / \mathbf{L})\end{array}$ & $\begin{array}{c}\text { Creatinine } \\
\text { (mg/dL) }\end{array}$ & $\begin{array}{c}\text { Total protein } \\
\text { (g/dL) }\end{array}$ \\
\hline 1 & Group I- Control (Normal saline 2ml kg p.o.) & $86.21 \pm 0.32$ & $7.51 \pm 0.21$ & $1.21 \pm 0.12$ & $6.44 \pm 0.15$ \\
\hline 2 & Group II (EEOS $200 \mathrm{mg} / \mathrm{kg} \mathrm{p.o)}$ & $88.35 \pm 0.12$ & $6.94 \pm 0.46$ & $1.35 \pm 0.09$ & $6.23 \pm 0.14$ \\
\hline 3 & Group III (EEOS $400 \mathrm{mg} / \mathrm{kg} \mathrm{p.o)}$ & $87.56 \pm 0.28$ & $7.02 \pm 0.51$ & $1.24 \pm 0.05$ & $6.26 \pm 0.49$ \\
\hline
\end{tabular}

Values are mean \pm SEM; $n=6$ in each group; Group -II \& Group III was compared with Group -I. The values of biochemical parameters rats were not altered significantly.

Table 10: Results of biochemical parameters of EEOS treated in rats

\begin{tabular}{|c|c|c|c|c|}
\hline SI. No & Animal treatment & Albumin (g/dL) & Globulin (g/dL) & Bilirubin (mg/dL) \\
\hline 1 & Group I- Control (Normal saline 2ml /kg p.o.) & $4.11 \pm 0.02$ & $4.73 \pm 0.11$ & $0.48 \pm 0.08$ \\
\hline 2 & Group II (EEOS 200 mg/kg p.o) & $4.58 \pm 0.12$ & $4.51 \pm 0.34$ & $0.56 \pm 0.10$ \\
\hline 3 & Group III (EEOS $400 \mathrm{mg} / \mathrm{kg} \mathrm{p.o)}$ & $4.21 \pm 0.32$ & $4.77 \pm 0.16$ & $0.51 \pm 0.21$ \\
\hline
\end{tabular}

Values are mean \pm SEM; $n=6$ in each group; Group -II \& Group III was compared with Group -I. The values of biochemical parameters rats were not altered significantly.

Table 11: Results of biochemical parameters of EEOS treated in rats

\begin{tabular}{|c|c|c|c|c|}
\hline SI. No & Animal treatment & SGPT (Units/L) & SGOT (Units/L) & ALP (Units/L) \\
\hline 1 & Group I- Control (Normal saline $2 \mathrm{ml} / \mathrm{kg} \mathrm{p.o.)}$ & $43.17 \pm 0.36$ & $121.3 \pm 0.21$ & $146.12 \pm 0.23$ \\
\hline 2 & Group II (EEOS $200 \mathrm{mg} / \mathrm{kg} \mathrm{p.o)}$ & $46.85 \pm 0.66$ & $126.4 \pm 0.34$ & $150.32 \pm 1.56$ \\
\hline 3 & Group III (EEOS $400 \mathrm{mg} / \mathrm{kg} \mathrm{p.o)}$ & $44.38 \pm 0.21$ & $120.2 \pm 0.05$ & $149.5 \pm 0.11$ \\
\hline
\end{tabular}

Values are mean \pm SEM; $n=6$ in each group; Group -II \& Group III was compared with Group -I. The values of biochemical parameters rats were not altered significantly.

Table 12: Results of biochemical parameters of EEOS treated in rats

\begin{tabular}{|c|c|c|c|}
\hline SI. No & Drug treatment & Cholesterol $(\mathbf{m g} / \mathbf{d L})$ & Triglycerides $(\mathbf{m g} / \mathbf{d L})$ \\
\hline 1 & Group I- Control (Normal saline $2 \mathrm{ml} \mathrm{kg} \mathrm{p.o.)}$ & $85.44 \pm 0.57$ & $44.12 \pm 0.42$ \\
\hline 2 & Group II (EEOS $200 \mathrm{mg} / \mathrm{kg} \mathrm{p.o)}$ & $82.27 \pm 0.58$ & $42.25 \pm 0.26$ \\
\hline 3 & Group III (EEOS $400 \mathrm{mg} / \mathrm{kg} \mathrm{p.o)}$ & $86.46 \pm 0.44$ & $43.11 \pm 0.26$ \\
\hline
\end{tabular}

Values are mean \pm SEM; $n=6$ in each group; Group -II \& Group III was compared with Group -I. The values of biochemical parameters rats were not altered significantly.

The results of glucose level in group -I were appearing in rats $86.21 \mathrm{mg} / \mathrm{dl}$. The treated animals like 200 and 400 $\mathrm{mg} / \mathrm{kg} /$ body weight were 88.35 and $87.56 \mathrm{mg} / \mathrm{dl}$ respectively. There is no elevation and significant changes were appeared when compared to Group- I. The result of urea in group-I were appear $7.51 \mathrm{mmol} / \mathrm{L}$. The treated group like 200 and $400 \mathrm{mg} / \mathrm{kg} /$ body weight were 6.94 and $7.02 \mathrm{mmol} / \mathrm{L}$ respectively. The results of glucose and urea were mentioned in table 9.

The creatinine level of control group was appeared $1.21 \mathrm{mg} / \mathrm{dL}$. The EEOS treated group 200 and $400 \mathrm{mg} / \mathrm{kg}$ were 1.35 and $1.24 \mathrm{mg} / \mathrm{dL}$ respectively. The total protein 
level of control group $6.44 \mathrm{~g} / \mathrm{dl}$ and the treated of EEOS 200 and $400 \mathrm{mg} / \mathrm{kg}$ were 623 and $6.26 \mathrm{mg} / \mathrm{dl}$ respectively. In Creatinine and total protein, there are no significant changes were report when compared to normal group. The results of Creatinine and total protein were mentioned in table 9.

The EEOS treated groups at 200 and $400 \mathrm{mg} / \mathrm{kg}$ p.o. did not show any significant changes in albumin, globulin and bilirubin when compared to Group-I. The results were shown in Table 10.

The ethanolic extracts of Oecophylla smaragdina (200 \& $400 \mathrm{mg} / \mathrm{kg}$ p.o respectively) treated groups were compared with Group-I. No significant changes were reported in SGPT, SGOT, ALP Cholesterol and Triglycerides. The results were shown in Table $11 \& 12$

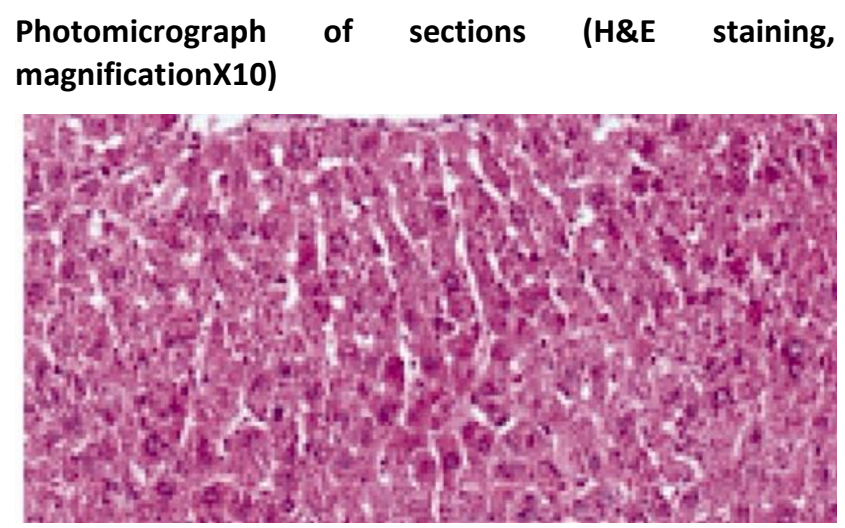

(a) Group I Control

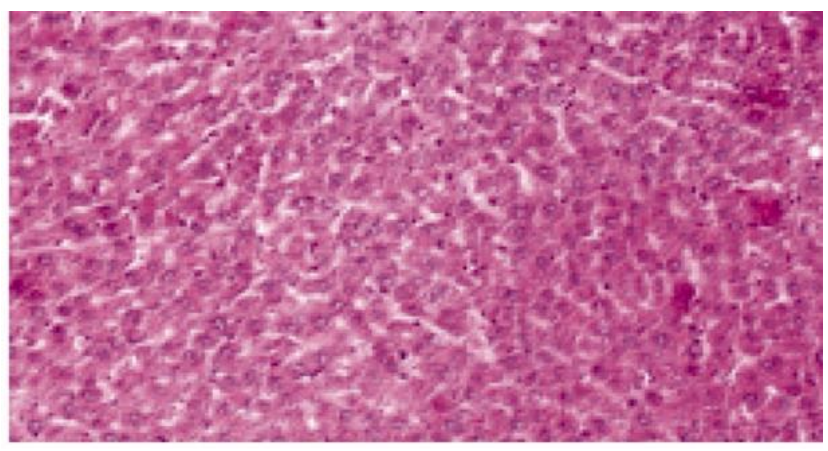

(b) Group II EEOS $200 \mathrm{mg} / \mathrm{kg}$ p.o.

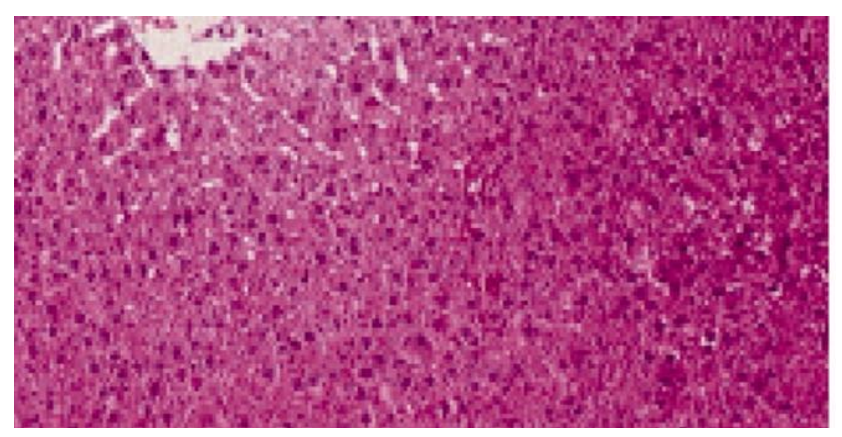

(c) Group III EEOS $400 \mathrm{mg} / \mathrm{kg}$ p.o.

Figure 1: Histopathological studies of rat liver treated with EEOS 200 and $400 \mathrm{mg} / \mathrm{kg} /$ oral

Control group, EEOS 200 and $400 \mathrm{mg} / \mathrm{kg}$ treated groups liver showed that normal hepatic cell has sinusoidal space, central vein showing normal architecture, absence of disarrangement, degeneration of hepatic cell, necrosis, sinusoidal haemorrhage and dilations. intact parenchymal cells and compactly arranged in the mucosal glands.

\section{CONCULSION}

The extractive value of EEOS was $8.56 \% \mathrm{w} / \mathrm{w}$. The preliminary chemical screening showed EEOS was rich in carbohydrates, free amino acids, alkaloids, lipid and steroids. The acute toxicity studies (as per OECD- 423 guidelines) results revealed that the $\mathrm{LD}_{50}$ values of EEOS was $2000 \mathrm{mg} / \mathrm{kg} /$ oral. The study revealed that there was no mortality up to the dose level of $2000 \mathrm{mg} / \mathrm{kg} /$ oral till the end of the study. Histopathological studies of the slides of cardiac muscles and liver of the animals treated with $2000 \mathrm{mg} / \mathrm{kg} /$ oral of EEOS showed normal architecture which confirms the safety of LD 50 doses.

EEOS was selected at two different dose levels of 200 and $400 \mathrm{mg} / \mathrm{kg}$ for the pharmacological screening in animals. The selected dose of EEOS at two different dose levels was subjected to gross behavioural studies in animals. The parameters such as spontaneous motor activity, effect on reflexes, effect on autonomic nervous system and effect after manipulations parameters proved that the EEOS at the above dose levels were safe and the animals were normal.

For investigating various parameters like organ weight, body weight, food intake, water intake and haematological/biochemical parameters on the animals treated with EEOS at the selected dose of 200 and $400 \mathrm{mg} / \mathrm{kg} /$ oral was given to rats for 28 days and using normal saline as control. Blood samples were collected and the following haematological parameters such as WBC count, RBC count, haemoglobin, lymphocytes, monocytes, were determined. Serum biochemical parameters such as glucose, cholesterol, creatinine, urea, triglycerides, bilirubin, total protein protein, albumin, globulin, ALP, AST and ALT were also determined. Histopathological studies on heart muscles and liver slices of the animals treated with the above extracts at the same dose level were studied. From the investigation various parameters like organ weight, body weight, food intake, water intake, haematological, serum biochemical and histopathological results, it has been observed that EEOS at 200 and $400 \mathrm{mg} / \mathrm{kg} /$ oral dose did not alter the above parameters when compared to control group animals.

Acknowledgement: The authors are thankful to Mr. S. Sreeram Ashok, Correspondent, Sankaralingam Bhuvaneswari College of Pharmacy, Sivakasi, Tamil Nadu for providing the facilities to carry out the research work.

\section{REFERENCES}

1. Efraim Lev. Traditional healing with animals (zootherapy): medieval to present-day Levantine practice, Journal of Ethnopharmacology, 2003; 85: 107-11

2. Adeola M O. Importance of wild Animals and their parts in the culture, religious festivals, and traditional medicine of 
Nigeria, Journal of Environmental Conservation, 1992; 19: $125-134$

3. Alves RN, Rosa IL, Santana GG. The role of animal-derived remedies as complementary medicine in Brazil, Journal of Biosciences, 2007; 57: 49-955.

4. Kunin WE and Lawton JH. Does biodiversity matter? Evaluating the case for conserving species. In: Gaston KJ (Ed), Biodiversity: a biology of num- bers and differences, Oxford: Blackwell Science, Springer, 1996; 1: 283-308.

5. Mirela Samfira1 and Ioan Petromanet. Therapeutic Value of the Human Being-Animal Relationship, Animal Science and Biotechnologies, 2011; 44 (2): 512-515

6. Anageletti, L.R., Agrimi, U., Curia, C., French, D., MarianiCostantini R. Healing rituals and sacred serpents. The Lancet, 1992; 340(8813): 223-225.

7. Akalesh Kumar Verma, Surya bali prasad, Thengtom Rongpi, Jashodeb Arjun). Traditional healing with animals (zootherapy) by the major ethnic group of karbianglong district of Assam, India, International Journal of Pharmacy and Pharmaceutical Sciences, 2014; 6(8): 593-600

8. Harborne, J.B. Textbook of Phytochemical Methods. A Guide to Modern Techniques of Plant Analysis. $5^{\text {th }}$ Edition, Chapman and Hall Ltd, London, 1998; 21-72.

9. Junping KOU, YunNi,Na Li, Jingrong Wang, Liang Liu, ZhiHong Jiang . Analgesic and Anti-inflammatory Activities of Total Extract and Individual Fractions of Chinese Medicinal Ants Polyrhachis lamellidens, Biological and Pharmaceutical Bulletin, 2005; 28(1): 176-180

10. Vinoth Prabhu V, Chidambaranathan N, Gopal. Evaluation of quantification of angiogenesis activity of Terminalia bellirica Roxy, by mice sponge implantation Method, Journal of Young Pharmacists, 2012; Vol.4(1): 22-27

11. OECD guidelines-423

12. OECD guidelines 407

13. Gerhard Vogel H. Drug Discovery and Evaluation, Springer, 2002; 2: 388-394.

Source of Support: None declared.

Conflict of Interest: None declared.

For any question relates to this article, please reach us at: editor@globalresearchonline.net New manuscripts for publication can be submitted at: submit@globalresearchonline.net and submit_ijpsrr@rediffmail.com 